Europhysics Letters

PREPRINT

\title{
Topological defects, pattern evolution, and hysteresis in thin magnetic films
}

\author{
P. A. Prudkovskit ${ }^{1}$, A. N. Rubtsov ${ }^{1}$ And M. I. Katsnelson ${ }^{2}$ \\ 1 Department of Physics, Moscow State University - 119992 Moscow, Russia \\ 2 Institute for Molecules and Materials, Radboud University Nijmegen - 6525 ED Ni- \\ jmegen, The Netherlands
}

PACS. 75.60. Ch - Domain walls and domain structure.

PACS. 75.60.Ej - Magnetization curves, hysteresis, Barkhausen and related effects.

PACS. 75.70.Ak - Magnetic properties of monolayers and thin films.

\begin{abstract}
Nature of the magnetic hysteresis for thin films is studied by the Monte-Carlo simulations. It is shown that a reconstruction of the magnetization pattern with external field occurs via the creation of vortex-antivortex pairs of a special kind at the boundaries of stripe domains. It is demonstrated that the symmetry of order parameter is of primary importance for this problem, in particular, the in-plane magnetic anisotropy is necessary for the hysteresis.
\end{abstract}

Introduction. - Traditional views assume that under the thermodynamic equilibrium conditions a system should either be homogeneous or consist of macroscopically large domains of homogeneous phases. It appears, however, that equilibrium or very long-lived metastable states occur frequently with a mesoscale heterogeneity (modulated phases, patterns, etc.; for a general review see, e.g., Ref. [1]). These inhomogeneous states may be either regular (e.g., stripes, stripe domains) or "chaotic". The stripes in high-temperature superconductors and doped Mott insulators [2,3], supramolecular self-assembly in organic chemistry [4], and "heterogeneous fluctuations" in metallic alloys [5] should be mentioned in this context. Stripe magnetic domain state of thin ferromagnetic films [6] under certain conditions turns out to be unstable with respect to the formation of complicated two-dimensional "chaotic" pattern [7-9], which provides another example of the mesoscale pattern evolution, interesting not only conceptually but also for the applications related to the information storage.

It is commonly accepted now that the formation of the mesoscale heterogeneity is a result of frustrations in the system which can result from either geometric factors $[5,10,11]$ or competing interactions, the long-ranged forces such as Coulomb or dipole-dipole interactions being of primary importance [3,7,12-14]. General concepts of "avoided criticality" [12,13] and "self-induced glassiness" [14] have been proposed to treat this situation. However, we still have no detailed theory to describe a formation of heterogeneous mesoscale states in frustrated systems. In particular, there is no satisfactory description of the experimentally observed $[8,9]$ "chaotization" of the magnetic stripe domains beyond the oversimplified Ising model; for the latter, several Monte-Carlo $[15,16]$ or phase-field [17] simulations have been

(C) EDP Sciences 
carried out. However, the vector character of the order parameter is of crucial importance for the magnetism.

In particular, the key issues for the applications such as the pattern evolution with the in-plane magnetic field variation and the magnetic hysteresis cannot be formulated in the framework of the Ising model. These questions are investigated in the present work.

Stripe domain formation is the result of an interplay of magnetostatic energy, energy of domain walls, and easy-axis magnetic anisotropy [6]. For the case of magnetic multilayers, the latter comes mainly from the layer interfaces. Easy magnetization axis normal to the film plane $x y$ appears [9]. Further we will argue that the in-plane anisotropy is also crucial for the magnetic behavior of the films. To demonstrate this we consider two limiting cases, that are the model with the two-component magnetization vector lying in the $y z$-plane and the three-component (Heisenberg) case without any $x y$-anisotropy.

Model and simulation results. - We start with the following effective Hamiltonian for the system under consideration:

$$
\begin{aligned}
& H=\int\left(\frac{J_{x}}{2}\left(\frac{\partial \mathbf{m}}{\partial x}\right)^{2}+\frac{J_{y}}{2}\left(\frac{\partial \mathbf{m}}{\partial y}\right)^{2}-\frac{K}{2} m_{z}^{2}-h m_{y}\right) d^{2} r+ \\
& +\frac{Q^{2}}{2} \iint m_{z}(\mathbf{r})\left(\frac{1}{\left|\mathbf{r}-\mathbf{r}^{\prime}\right|}-\frac{1}{\sqrt{d^{2}+\left(\mathbf{r}-\mathbf{r}^{\prime}\right)^{2}}}\right) m_{z}\left(\mathbf{r}^{\prime}\right) d^{2} r d^{2} r^{\prime}
\end{aligned}
$$

Here $\mathbf{m}$ is the magnetization unit vector having either two or three components, $h$ is an external field, $J_{x}, J_{y}, K$ are exchange and anisotropy parameters, and the last term describes the long-range dipole-dipole interaction [7] $(Q$ is the effective magnetic charge density and $d$ is the film thickness). Note that stripes appear only if the exchange interaction is anisotropic; a checkerboard-like structure arises for $J_{x}=J_{y}[9]$. With our choice of parameters, stripes are formed along $y$-axis, that is parallel to the external field. The domain structure arises for not too strong external fields since at $h \rightarrow \infty$ the magnetization vector is just parallel to the $y$-axis. We will consider the case of a regular stripe domain structure is stable at zero external field (which is observed, e.g., for the permalloy-cobalt multilayers in Ref. [9]). In these experiments an instability of the stripe domain structure with the external field increase has been observed, which can be described in terms of the appearance of disclinations at the domain wall lines $[8,18]$; further a two-dimensional "chaotic" magnetization pattern is formed.

In our Metropolis Monte Carlo [19] simulations, a discrete-lattice (300 by 300 sites) analogous to the effective Hamiltonian (11) is studied, in particular, the exchange terms are approximated by appropriate nearest-neighbor interactions. We present here the data for the system with $J_{x}=2, J_{y}=4, K=4, Q^{2}=0.1$ and $d=10$ obtained at several values of the temperature. The parameters are chosen to produce the stripe domains with a width $\Lambda$ of about 10 lattice periods. In each simulation, we start with a large (saturating) negative external field and slowly varied it to a large positive value and then back.

Figure 1 shows the magnetization curves $M_{y}(h)$ for several values of temperature for the two-component $(y z)$ magnetization. Well-pronounced hysteresis loops are clearly seen. To reveal the source of the hysteresis, we display snapshots of the system at several points of the hysteresis loop (Figure 21). Inside the stripe domains, the magnetization is oriented almost perpendicular to the film plane, so that $m_{z}=1$ and $m_{z}=-1$. These areas are drawn by red and light-blue colors, respectively. At a domain wall, the magnetization has to pass through the points where $m_{y}=-1$ or $m_{y}=1$. These points are shown by and dark-blue and yellowgreen colors. Mixtures or the appropriate colors is used for intermediate orientations of the magnetization. 


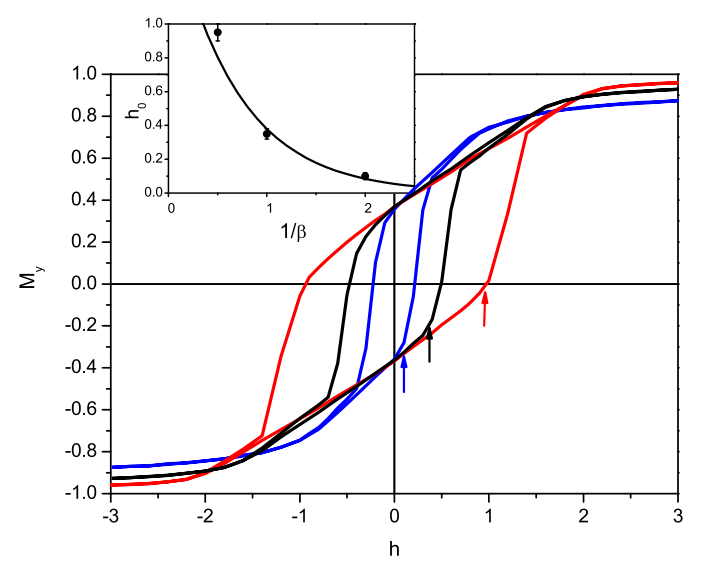

Fig. 1 - Magnetization curves for the system with two-component magnetization. Red, black and blue loops correspond to the temperature values $1 / \beta=0.5,1$ and 2 , respectively; other parameters are given in the text. Arrows indicate the critical field, $h$, corresponding to the nucleation of the vortex-antivortex pairs. The inset shows these values of $h$, whereas the line in the inset shows the estimation (3) with $\ln \frac{T L}{t_{0} \sigma}=13$

At zero field, the magnetization rotates through the negative $m_{y}$-direction for all domain walls. At a certain positive $h$, "green" parts of the domain walls with positive $m_{y}$ arise. Further increase of the external field results in a sudden flip of all domain walls to positive $m_{y}$. One can see that the magnetization vector rotates over $2 \pi$ angle at the motion along a closed loop containing the end point of the "green" part. Thus, the magnetization flip regions of the domain walls are terminated by vortex-antivortex pairs. It should be stressed that here both the vortex and antivortex always locate at the same linear domain wall contrary to the Kosterlitz-Thouless situation [20] where the vortices can travel in the entire plane.

Similar computations have been performed for the case of three-component magnetization (Heisenberg ferromagnet with dipole-dipole interactions). The system has a similar stripe domain structure. However, in the very contrast to the two-component case, no hysteresis is observed within the errorbar of calculation, as Figure 3 shows.

Physical picture. - Let us first discuss the source of hysteresis for the two-component magnetization. Consider the energy balance of a single vortex-antivortex pair starting with the case $h=0$. First of all, creation of the vortex itself requires a finite energy. Near the vortex singularity, energy is mainly contributed by the exchange (gradient) terms in Eq. (10), similarly to XY-model [20]. The size of this area can be estimated as a smaller value from the vortex-antivortex distance $l$ and domain wall thickness $\sigma=\sqrt{J_{x} / K}$. A standard estimation with the logarithmic accuracy similar to Ref. [20] results in the expression for the energy of pair at $h=0$ :

$$
\begin{gathered}
E(l<\sigma)=2 \pi \sqrt{J_{x} J_{y}} \ln (l / r), \\
E(l>\sigma)=2 \pi \sqrt{J_{x} J_{y}} \ln (\sigma / r)
\end{gathered}
$$

Here the short-range cutoff of order of the lattice constant, $r_{0}$, is introduced. 

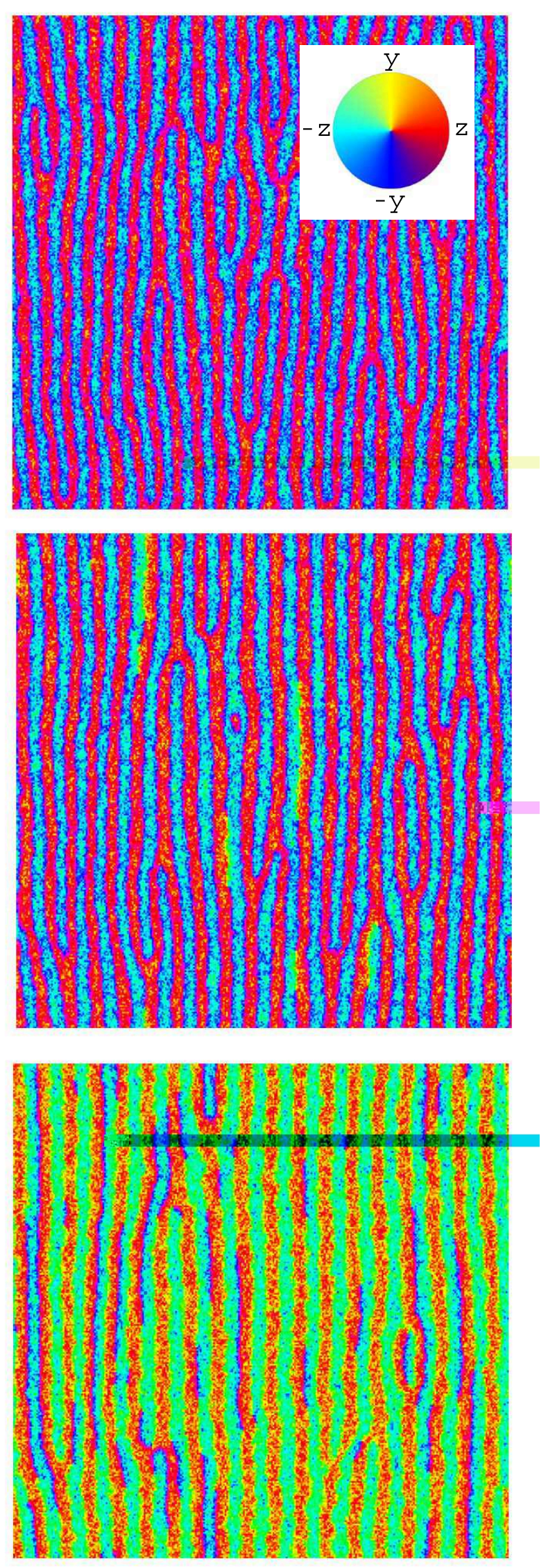

Fig. 2 - Snapshots of the stripe-domain system with the two-component order parameter at several points of the hysteresis loop for $\beta=1$. Magnetic field is $h=0, h=0.3$, and $h=0.6$, from top to bottom shot. The inset shows the color legend for the orientation of local magnetization. 


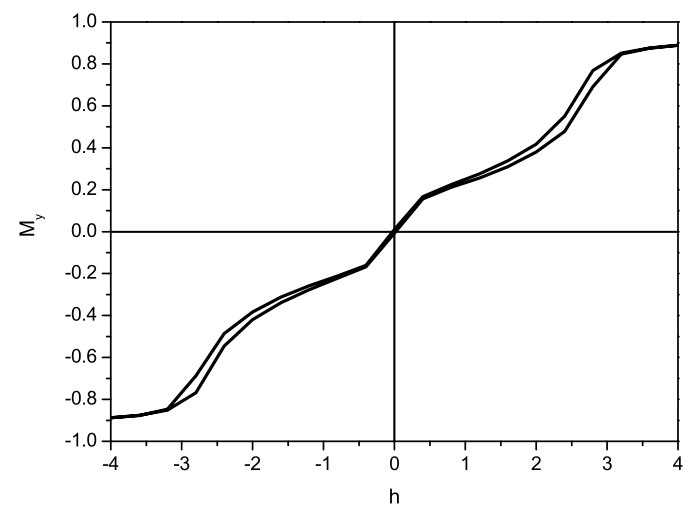

Fig. 3 - Magnetization curve for the case of tree-component magnetization at temperature $1 / \beta=1$. All the parameters are the same as for $1 / \beta=1$ magnetization curve in Figure 1.

The existence of hysteresis means that the value of $E(l>\sigma)$ is large enough in comparison with the temperature, otherwise thermal fluctuations should destroy oriented domain walls. The external field makes the wall flip energetically favorable and decreases the barrier between the the flipped and unflipped wall. Let us estimate the height of this barrier. We will discuss the lower part of the magnetization curve, so that positive $h$ tends to flip the domains. Consider the term $-h m_{y}$ as a perturbation. The zero-order approximation for $\mathbf{m}(\mathbf{r})$ can be constructed explicitly for $l \ll \sigma$, because depolarization effects and the anisotropy term in (11) can be neglected in this case. The first-order correction to $E(l \ll \sigma)$ appears to be $h\left(\int m_{y} d^{2} r\right)_{h=0}=-\pi \ln 2 \sqrt{J_{x} J_{y}} l^{2} h$. For large $l$, this correction is obviously proportional to $l$, that is $E(l \gg \sigma) \propto-l h / \sigma$. One can see that the function $E(l)$ at non-zero positive $h$ has a maximum at $l \ll \sigma$ or $l \sim \sigma$. Use the limit of small $l$ for the logarithmic-accuracy estimation. We obtain that the maximum of $E(l)$ take place at $l_{\max }^{2} \propto J_{x} / h$ and $E\left(l_{\max }\right) \approx \pi \sqrt{J_{x} J_{y}} \ln \frac{J_{x}}{r_{0}^{2} h}$. There is a noticeable probability of the vortex-antivortex pair nucleation at the domain wall interval of the length $L$ during the time $T$, if $e^{-\beta E\left(l_{\max }\right)} \approx \frac{T L}{t_{0} \sigma}$, where short-time cut-off $t_{0}$ is the shortest relaxation time of the system [21]. Therefore we estimate that the vortexantivortex pairs arise at

$$
h \propto \frac{J_{x}}{r_{0}^{2}} \exp \left(-\frac{\ln \frac{T L}{t_{0} \sigma}}{\pi \beta \sqrt{J_{x} J_{y}}}\right) .
$$

It is worthwhile to note at this point that, strictly speaking, the applicability of the Monte Carlo scheme is proven only for the thermodynamic equilibrium case. However, it is clear that for the situation of several well-separated local minima, individual properties of those metastable states are also described correctly. The transition through energy barrier between the minima in the Metropolis Monte Carlo simulation is in fact a diffusion process with a typical timescale $t_{0}$ of about a single Monte Carlo sweep, therefore in our numerical calculations $\ln \frac{T L}{t_{0} \sigma} \approx 10 \div 15$. The obtained dependence agrees well with the numerical data, as the inset in Fig. 1 shows.

Possible effects of interaction between domain walls should be taken into account. To determine this interaction, it is sufficient to consider only the structure which is homogeneous 
in the $y$-direction. One should note first that the domain wall flip does not affect the dipoledipole interaction term since this flip does not change $z$-component of the internal magnetic field. Therefore we can ignore the last term in Eq.(11) in our estimation. At zero external field, Eq. (11) reads

$$
\int\left(\frac{J_{x}}{2} \dot{\phi}^{2}-\frac{K}{2} \sin ^{2} \phi\right) d x=\min
$$

where the phase $\phi$ describing the orientation of two-component magnetization is introduced: $\mathbf{m}=(\sin \phi, 0, \cos \phi)$. This is equivalent to the minimal action condition for a pendulum, if $x$ acts as a time [22]. Qualitatively, the domain wall corresponds to a potential energy minimum of the pendulum $\phi=0, \pm \pi, \ldots$, whereas domains themselves are described by the near-separatrix motion $\phi \approx \pm \pi / 2, \pm 3 \pi / 2, \ldots$. Domains with parallel and antiparallel walls are described by the conditions $\phi(0)=0, \phi(\Lambda)=0$ and $\phi(0)=0, \phi(\Lambda)=\pi$, respectively. The difference between the values of the action for these two trajectories gives an effective interaction energy of domain walls. For the case of $\Lambda / \sigma \gg 1$, the parallel wall configuration is energetically favorable, but the energy gain is exponentially small with a factor of $e^{-\Lambda / \sigma}$. In our simulations as well as in the experiment [9] $\Lambda / \sigma \approx 7 \div 10$, and this exponentially small term can be neglected. It would be interesting to realize experimentally a system with $\Lambda \approx \sigma$, where the interaction between domain walls would be important.

In the case of the Heisenberg model (three-dimensional vector order parameter without $x y$ anisotropy), the vortices and antivortices will not arise since the orientations of domain walls can be changed without a transition over an energy barrier. For example, for the domain profile passing from $m_{z}=1$ to $m_{z}=-1$ orientation via $y$-direction, one can consider the $180^{0}$ rotation of $\mathbf{m}$ in the $x y$-plane. The magnetization distribution $m_{x}=\sin \phi \sqrt{1-m_{z}^{2}}, m_{y}=$ $\cos \phi \sqrt{1-m_{z}^{2}}$ interpolates smoothly between the domain walls passed through $+y$ and $-y$ $(\phi=0$ and $\phi=\pi)$ with the energy independent on $\phi$, as one can see from Eq. (11). In agreement with these simple topological considerations, our numerical simulations do not show any hysteresis effects for the Heisenberg case. This demonstrates the relevance of in-plane magnetic anisotropy for the hysteresis in magnetic films and multilayers. This anisotropy can suppress a continuous rotation of the magnetization vector and therefore result in a hysteresis behavior.

We investigate here a simplified model of the stripe-domain formation; in the conclusion, we discuss its relation with real experimental situation. Although the Hamiltonian (1) is frequently used to describe the stripe domains [7], it may by a concern that the dipole-dipole interactions due to the in-plane magnetization components are neglected in this approach. Indeed, the effective density of magnetic charges is $-Q$ div $\mathbf{m}$, and only the surface charges due to the discontinuity of the normal component of $\mathbf{m}$ at the film boundary are taken into account in Eq.(11). The bulk charge density is proportional to $\frac{\partial m_{x}}{\partial x}+\frac{\partial m_{y}}{\partial y}$ which is zero inside the domains but not in the domain walls. Physically, the in-plane dipole moment of the domain wall may occur. The detailed study of these effects is out of the scope of the paper; let us only justify why they can be neglected in the cases under consideration. First of all, the bulk magnetic charges do not appear for the two-component order parameter since $m_{x}$ is absent and $m_{y}$ is independent of $y$. For the three-component order parameter, the domain wall may have a dipole moment of order of $Q \sigma d$ per unit wall length. This value should be compared with similar quantity of the domain, that is $Q \Lambda d$. Therefore our consideration is valid assuming that $\sigma \ll \Lambda$. Qualitatively, even in this case the bulk charges introduce some anisotropy in the $x y$-plane and can therefore result in hysteresis effects for the three-component magnetization, but these effects should be much weaker than for the two-component case. 
P. A. Prudkovskit, A. N. Rubtsov and M. I. Katsnelson: Topological Defects, Pattern evolution, And hysteresis in t

The work was supported by the Netherlands Organization for Scientific Research (NWO project 047.016.005). One of the authors (A.R.) is grateful to the "Dynasty" foundation for the support.

\section{REFERENCES}

[1] Seul M. and Andelman D., Science, 267 (1995) 476.

[2] Tranquada J. M., Sternlieb B. J., Axe J. D., Nakamura Y., and Uchida S., Nature (London), 375 (1995) 561.

[3] Emery V. J. and Kivelson S. A., Physica C, 209 (1993) 597.

[4] Supramolecular Chemistry and Self-Assemlby. Special Issue: Science, 295 (2002) 2400-2421.

[5] Gornostyrev Yu. N., Katsnelson M. I., and Trefilov A. V., J. Phys.: Condens. Matter, 9 (1997) 7837.

[6] Kittel C.,, Phys. Rev. 70, 965 (1946).

[7] Garel T. and Doniach S., Phys. Rev. B, 26 (1982) 325.

[8] Seul M. and Wolfe R., Phys. Rev. Lett., 68 (1992) 2460.

[9] Brucas R., Hafermann H., Katsnelson M. I., Soroka I. L., Eriksson O., and Huorvarsson B., Phys. Rev. B, 69 (2004) 064411.

[10] Kleman M., Adv. Phys., 38 (1989) 605.

[11] Nelson D. R.,, Defects and Geometry in Condensed Matter Physics (Cambridge Univ. Press, Cambridge) 2002.

[12] Kivelson D., Kivelson S. A., Zhao X., Nussinov Z., and Taruus G., Physica A, 219 (1995) 27.

[13] Nussinov Z., Rudnick J., Kivelson S. A., and Chayes L. N., Phys. Rev. Lett., 83 (1999) 472.

[14] Schmalian J., and P. G. Wolynes, Phys. Rev. Lett., 85 (2000) 836.

[15] Bоотh I., Macisaac A. B., Whitehead J. P., and De'Bell K., Phys. Rev. Lett., 75 (1995) 950.

[16] Grousson M., Tarjus G., and Viot P., Phys. Rev. E, 64 (2001) 036109.

[17] Jagla E. A., Phys. Rev. E, 70 (2004) 046204.

[18] Kashuba A. B. and Pokrovsky V. L., Phys. Rev. B, 48 (1993) 10335.

[19] Landau D. P. and Binder K., A Guide to Monte Carlo Simulations in Statistical Physics (Cambridge Univ. Press, Cambridge) 2000.

[20] Kosterlitz J. M. and Thouless D. J., J. Phys. C, 6 (1973) 1181.

[21] Kramers H. A., Physica (Utrecht), 7 (1940) 284.

[22] Goldstein H., Classical Mechanics (Addison-Wesley, Reading, MA) 1980. 